\title{
The role of tumor suppressing microRNA let-7 in undifferentiated nasopharyngeal carcinoma
}

TS Wong, W Gao, JZH Li, JYW Chan. The University of Hong Kong, Hong Kong, Hong Kong

AIMS:This study aimed at evaluating the potential anti-proliferative effects of the microRNA let-7 family in nasopharyngeal carcinoma (NPC) cells. In addition, the association between let-7 suppression and DNA hypermethylation is examined. MATERIALS AND METHODS:Levels of mature let-7 family members (-a, -b, -d, -e, -g, and -i) in normal nasopharyngeal cells (NP69 and NP460) and nasopharyngeal carcinoma cells (HK1 and HONE1) were measured by real-time quantitative PCR. Cell-proliferation assay and c-Myc immunohistochemical staining were performed on NPC cells transfected with let-7 precursor molecules. In addition, expression changes in let-7 family members in response to demethylating agents (5-azacytidine and zebularine) were also examined. RESULTS:In comparison with the normal nasopharyngeal cells, let-7 (-a, -b, -d, $\mathrm{e},-\mathrm{g}$, and -i) levels were reduced in nasopharyngeal carcinoma cells. Ectopic expression of the let-7 family in nasopharyngeal carcinoma cells resulted in inhibition of cell proliferation through downregulation of c-Myc expression. Demethylation treatment of nasopharyngeal carcinoma cells caused activation of let-7 expression in poorly differentiated nasopharyngeal carcinoma cells only. CONCLUSION:Our results suggested that miRNA let-7 might play a role in the proliferation of NPC. DNA methylation is a potential regulatory pathway, which is affected when let-7 is suppressed in NPC cells. However, the extent of DNA hypermethylation/hypomethylation in regulating let-7 expression requires further elucidation. 